\title{
Desarrollo inicial de plantas de Mimosa scabrella bajo diferentes regímenes hídricos
}

\author{
Initial development of Mimosa scabrella seedlings under different water regimes
}

\author{
Eduarda Demari Avrella a*, Luciana Pinto Paim a, Edgar Aaron Rodriguez Rivera a, \\ Michael Mazzurana ${ }^{\text {}}$, Gilmar Schafer ${ }^{\text {a }}$, Claudimar Sidnei Fior ${ }^{\text {a }}$ \\ *Autor de correspondencia: ${ }^{a}$ Universidade Federal do Rio Grande do Sul, Faculdade de Agronomia \\ Departamento de Horticultura e Silvicultura, Avenida Bento Gonçalves, 7712, Porto Alegre, Rio Grande do Sul, Brasil, \\ tel.: +55 05198040-5501, dudademari@hotmail.com, lucianappaim@bol.com.br, edgar.rivero@ufrgs.br, schafer@ufrgs.br, \\ csfior@ufrgs.br \\ ${ }^{\mathrm{b}}$ Universidade Federal do Rio Grande do Sul, Faculdade de Agronomia, Departamento de Solos, Porto Alegre, Brasil, \\ michael.mazurana@gmail.com
}

\section{SUMMARY}

One of the main abiotic factors that influence the growth and development of plants is water, and consequently the use of water stress-tolerant species increases the chances of success of crops in these conditions. The aim of this study was to evaluate the growth and initial development of Mimosa scabrella seedlings under different water regimes. Therefore, seeds were submitted to dormancy breaking and sowed in coir fiber substrate. After that, the seedlings were established in pots ( $8 \mathrm{~L}$ capacity) containing composted pine bark substrate. The treatments consisted of five levels of pot capacity: $100 \%, 75 \%, 50 \%, 25 \%$ and $10 \%$. The experimental design was completely randomized with four replicates of five plants per treatment. There was influence of water regimes in most of the variables analyzed, where it was observed that with $50 \%$ of pot capacity growth and development of plants showed satisfactory results. Moreover, the quality of the seedlings showed that in general, with only $25 \%$ of the pot capacity plants have the ability to survive and settle under water stress conditions. Thus, the data indicate that Mimosa scabrella is a promising species for cultivation under water deficit, since the growth and initial development of seedlings occurred satisfactorily in conditions similar to these.

Key words: hydric stress, native species, seedling quality, substrate, bracatinga.

\section{RESUMEN}

El agua es uno de los principales factores abióticos que influyen en el crecimiento y desarrollo de las plantas; la utilización de especies más tolerantes al déficit hídrico aumenta las oportunidades de éxito de los cultivos en estas condiciones. El objetivo de este estudio fue evaluar el crecimiento y desarrollo inicial en plantines de Mimosa scabrella bajo diferentes regímenes hídricos. Con ese fin, un número determinado de semillas fueron sometidas a la superación de la latencia y sembradas en sustrato a base de fibra de coco. Posteriormente, las plantas fueron colocadas en macetas (capacidad de ocho litros), con sustrato de corteza compostada de pino. Los tratamientos fueron definidos por cinco niveles de contenido de agua: $100 \%, 75 \%, 50 \%, 25 \%$ y $10 \%$. Se utilizó un diseño completamente al azar con cuatro repeticiones de cinco plantas por tratamiento. Como resultado, hubo influencia de los regímenes hídricos en la mayoría de las variables analizadas, en las cuales se observó que hasta en $50 \%$ de contenido de agua, el crecimiento y el desarrollo de las plantas presentaron resultados satisfactorios. La calidad de los plantines demostró, que en general, en un $25 \%$ de contenido de agua las plantas presentan capacidad de sobrevivir y de establecerse en condiciones de estrés hídrico. Así, los datos apuntan que $M$. scabrella es una especie potencial para el cultivo bajo condiciones de estrés hídrico, pues el crecimiento y desarrollo inicial de los brinzales ocurrieron de forma satisfactoria en condiciones análogas a estas.

Palabras clave: estrés hídrico, especie nativa do Brasil, calidad de plantines, sustrato, bracatinga.

\section{INTRODUCCIÓN}

La implantación de asentamientos forestales de uso múltiple, tales como la producción de madera y aceites esenciales, constituye una alternativa viable para la reducción de la presión ejercida sobre los bosques nativos, ocasionada principalmente por el consumo de productos derivados de la madera que ponen en riesgo la extinción de varias especies vegetales (Lenhard et al. 2010). Un ejemplo de eso es la bracatinga (Mimosa scabrella Benth.), que puede constituir una importante especie alternativa de la región sur de Brasil (Hoffer et al. 2015, Menegatti et al. 2016), debido a que tiene características relevantes para la exploración de múltiples productos $\mathrm{y}$, además, por la importancia ambiental al ser una especie que ayuda en los procesos de regeneración de la vegetación en áreas degradadas. 
La bracatinga es una especie típica de la meseta surbrasileña que se encuentra en la vegetación secundaria de la Selva Ombrófila Mista (Machado et al. 2008), con distribución geográfica desde el sur de Minas Gerais y Río de Janeiro, hasta el norte de Río Grande del Sur, donde se encuentra con mayor frecuencia (Saueressig 2014). Es una especie leguminosa pionera, de ciclo corto, que se destaca por un rápido crecimiento, alcanzando los $20 \mathrm{~m}$ de altura con un diámetro a la altura del pecho de $40 \mathrm{~cm}$, después de ocho años de edad (Fabrowski et al. 2005). El principal uso es de madera para la generación de energía. Por tanto, la especie es bastante versátil ya que puede ser utilizada para producción de madera, celulosa y paneles, como facilitadora para ambientes degradados, con potencial ornamental, forrajero, melífero y medicinal (Saueressig 2014).

$\mathrm{Su}$ crecimiento y desarrollo vegetal ocurre en ambientes compuestos por varios factores abióticos que varían en el tiempo y espacio. La fluctuación de esos factores fuera de sus límites normales puede provocar alteraciones morfológicas en las plantas (Menegatti et al. 2017). El agua es uno de los principales factores que influyen en el crecimiento y desarrollo de las plantas debido a que es el componente de mayor porción del contenido celular (Taiz y Zeiger 2013). El estrés hídrico provoca alteraciones en el comportamiento vegetal, cuya irreversibilidad dependerá del genotipo, de la duración, de la severidad y de la etapa de desarrollo de las plantas (Santos y Carlesso 1998). De esta manera, la respuesta de muchas plantas a la sequía consiste en el aumento de la actividad del ácido abscísico, disminución en la apertura y densidad estomática, el crecimiento de la biomasa aérea, acumulación de solutos compatibles para mantener la turgencia y acelerar la senescencia, así como la abscisión de las hojas más viejas (Smirnoff 2014).

La disminución de la turgencia y del crecimiento de las plantas son las dos respuestas más sensibles al estrés hídrico. De esta manera, cuando las células vegetales sufren estrés hídrico, ocurre alteración de la extensibilidad de la pared y disminución en el índice de división celular, lo que puede afectar los procesos fisiológicos básicos, no obstante, un efecto secundario de esta deshidratación celular es la mayor concentración de iones, pudiendo alcanzar niveles tóxicos (Claeys y Inzé 2013).

El déficit hídrico restringe el crecimiento de las plantas, de tal modo que la productividad de la biomasa pasa a expresar solamente una fracción de su potencial genético (Verslues et al. 2006). De esta forma, a la medida que la planta tolera este estrés, ella se aclimata, lo que es el resultado de eventos íntegros que ocurren en todos los niveles de organización, como anatómico, morfológico, bioquímico y fisiológico (Lopes et al. 2011). Por lo tanto, la sensibilidad de las plantas a estos procesos es, en gran parte, el reflejo de una estrategia para liderar con la fracción de variación de disponibilidad de agua (Smirnoff 2014).

El uso de especies nativas de lugares específicos, adaptadas a las severas condiciones climáticas de su región de origen, aumenta las probabilidades de éxito en una reforestación, además de constituir una importante alternativa para el aprovechamiento de las áreas degradadas, ya que en estas condiciones el cultivo de muchas especies agrícolas es inviable económicamente (Silva et al. 2009). Basándose en esto, se plantea como hipótesis de trabajo que las plantas de bracatinga sometidas a condiciones de estrés hídrico presentan características que indican su capacidad de sobrevivir y de establecerse en campo con condiciones similares a estas. Por lo tanto, el objetivo de este estudio es evaluar el crecimiento y el desarrollo inicial de plantines de Mimosa scabrella Benth. bajo diferentes regímenes hídricos, simulados a través de cinco niveles de contenido de agua.

\section{MÉTODOS}

El presente estudio fue realizado en condiciones controladas bajo invernadero, perteneciente al Departamento de Horticultura y Silvicultura de la Universidad Federal de Río Grande del Sur, en Porto Alegre, RS (Brasil), durante el período comprendido desde octubre de 2014 a marzo de 2015 (con temperaturas medias de máxima de $38^{\circ} \mathrm{C}$ y mínima de $20^{\circ} \mathrm{C}$, durante el período de conducción del experimento, obtenido a partir de un termómetro digital con almacenamiento de datos). Las semillas fueron recolectadas de ocho árboles madre en el municipio de Santo Antonio do Palma, RS., en diciembre de 2012 (ejemplar registrado en el herbario de la UFRGS, ICN 184890), y almacenadas en refrigerador $\left(\mathrm{a} 5^{\circ} \mathrm{C}\right)$ por aproximadamente dos años.

Con el propósito de superar la latencia, las semillas fueron remojadas en agua a $90{ }^{\circ} \mathrm{C}$ hasta alcanzar temperatura ambiente. Este tratamiento fue seleccionado de un estudio piloto como el más práctico y rápido para el rompimiento de la latencia de las semillas de bracatinga. Posteriormente fueron sembradas en bandejas de poliestireno expandido conteniendo sustrato a base de fibra de coco (pó-de-coco Golden Mix tipo PM $^{\circledR}$-AMAFIBRA), sembrando tres semillas por cada alvéolo. Cuando las plántulas alcanzaron aproximadamente $5 \mathrm{~cm}$ de altura $(\sim 20$ días después de la siembra) se realizó el raleo, manteniendo solamente una plántula, la que presentaba más vigor. Después de 43 días se realizó el trasplante a macetas con una capacidad de ocho litros, conteniendo como sustrato corteza compostada de pino, cuyas principales características químicas y físicas se presentan en el cuadro 1 .

Los tratamientos fueron definidos por cinco niveles de contenido de agua ( $100 \%, 75 \%, 50 \%, 25 \%$ y $10 \%$ ), refiriéndose a $100 \%$ de saturación del sustrato, los cuales fueron determinados a través de análisis en el Laboratorio de Análisis de Sustratos del Departamento de Horticultura y Silvicultura de la Facultad de Agronomía, UFRGS. A partir de esto, se calcularon las cantidades de agua necesarias para la primera irrigación de todas las unidades experimentales, a fin de hidratar el sustrato de forma equivalente a la condición de capacidad de la maceta de cada tratamiento. 
Cuadro 1. Propiedades químicas y físicas del sustrato corteza compostada de pino.

Chemical and physical properties of the substrate composted pine bark.

\begin{tabular}{lc}
\hline \multicolumn{2}{c}{ Propiedades químicas } \\
\hline $\mathrm{CE} 1: 5\left(\mathrm{dS} \mathrm{m}^{-1}\right)$ & 0,51 \\
$\mathrm{pH}\left(\mathrm{H}_{2} \mathrm{O}\right)$ & 6,69 \\
\hline \multicolumn{2}{c}{ Propiedades físicas } \\
\hline Densidad seca $\left(\mathrm{kg} \mathrm{m}^{-3}\right)$ & 438,9 \\
Porosidad total $(\%)$ & 64,70 \\
Espacio de aireación (\%) & 20,23 \\
Agua fácilmente asimilable (\%) & 10,31 \\
Agua de reserva (\%) & 1,66 \\
Agua difícilmente disponible (\%) & 32,50 \\
\hline
\end{tabular}

Los recipientes del tratamiento $100 \%$ tuvieron en sus bases platos con altura de $1,5 \mathrm{~cm}$, los cuales permanecieron con agua para garantizar la completa hidratación del sustrato a tiempo completo. Con base en esto, fue posible calcular diariamente la cantidad de agua correspondiente a cada tratamiento, por la cantidad de agua evaporada del plato de un día para el otro. Por ejemplo, cuando la necesidad de reposición de agua en el plato de la maceta $100 \%$ era de $100 \mathrm{~mL}$, en los demás se añadían 75, 50, 25 y $10 \mathrm{~mL}$, para los tratamientos $75,50,25$ y $10 \%$, respectivamente.

Se realizó la fertilización a los 15,76 y 114 días después del inicio del experimento, a través de la incorporación al sustrato de $8 \mathrm{~g} \mathrm{~L}^{-1}$ de una fertilización de base compuesta por fertilizante granulado NPK (nitrógeno, fósforo y potasio), formulación 7:11:9.

Las evaluaciones se realizaron cada 15 días en las variables altura y diámetro del cuello. La altura fue obtenida por medio de una regla milimétrica, medida desde el cuello de la planta hasta la yema apical y el diámetro del cuello con un pie de metro digital. Después de haber transcurrido 126 días desde el trasplante, fueron evaluadas las variables: altura, diámetro del cuello, biomasa fresca y seca (aérea y radical), a través de la diferencia porcentual del peso después de ser secada a $65^{\circ} \mathrm{C}$ hasta peso constante, y el volumen total de las raíces, a través de la diferencia de volumen en probeta graduada. Con base en los datos, se realizó la evaluación de la calidad de los plantines a través del índice de calidad de Dickson (ICD) (Dickson et al. 1960), de la relación altura y biomasa seca aérea (H/BSPA) y de la relación biomasa seca aérea y radical (BSPA/BSR), de acuerdo con Gomes y Paiva (2013).

Se utilizó un diseño completamente al azar con cuatro repeticiones, cinco plantas por unidad experimental, totalizando 100 plantas. Las variables altura, diámetro del cuello y relación masa seca de la parte aérea y del sistema radical fueron sometidos al análisis de varianza (ANOVA) por medio del programa estadístico Costat 6.4. Con el programa SigmaPlot 11.0 se realizó una regresión polinomial. Por otra parte, las variables biomasa fresca y biomasa seca (aérea y radical), volumen total de raíces, índice de calidad de Dickson y relación altura y masa seca de la parte aérea, fueron sometidos al análisis no paramétrico por la prueba de Kruskal-Wallis y regresión no lineal, pues no atendieron a los supuestos del análisis de varianza paramétrica, incluso después de las transformaciones de los datos.

\section{RESULTADOS}

Se observaron diferencias significativas en el crecimiento y desarrollo inicial de los plantines en función de los diferentes niveles de déficit hídrico en la irrigación del sustrato, con excepción de la variable relación masa seca de la parte aérea y del sistema radical $(P=0,14)$.

Los niveles de déficit hídrico influenciaron de forma proporcional el desarrollo inicial de los plantines de bracatinga, pues con la reducción de la disponibilidad hídrica hubo disminución en el crecimiento en las plantas. Para la altura y el diámetro del cuello, este descenso presentó tendencia exponencial inversamente proporcional al aumento del déficit hídrico (figura 1A y B), donde se observó una reducción más acentuada a partir del contenido de agua de $50 \%$.

La mayor altura fue observada en el tratamiento con contenido de agua de $100 \%(126,56 \mathrm{~cm})$, ocurriendo una reducción de apenas 3,45\% para el tratamiento $75 \%$ y $22,36 \%$ para el tratamiento $50 \%$. A partir de ahí, hubo una reducción drástica de 63,09 y 90,52\% para los tratamientos de 25 y $10 \%$, respectivamente. Los datos referentes al diámetro del cuello de las plantas presentaron resultados similares, es decir, hubo una baja reducción en niveles con menor déficit hídrico $(8,31$ y $28,74 \%$ para los tratamientos de 75 y $50 \%$, respectivamente) y decrecimiento acentuado en los tratamientos del $25 \%(62,30 \%)$ y el $10 \%(86,58 \%)$ de contenido de agua en comparación con el tratamiento del $100 \%$.

El crecimiento de las plantas en la altura y diámetro del cuello a lo largo del tiempo del ensayo presentó un aumento cuadrático para los tratamientos de 100,75 y $50 \%$ de contenido de agua y aumento con tendencia lineal para los tratamientos con mayor déficit hídrico (figura $1 \mathrm{C}$ y D). Con los menores niveles de déficit hídrico (100, 75 y $50 \%$ de la capacidad de la maceta) se obtuvieron resultados superiores para estas dos variables.

Los resultados de la biomasa fresca y seca de la parte aérea presentaron un descenso sigmoidal con la reducción de la disponibilidad hídrica, donde a partir del $75 \%$ de contenido de agua hubo una caída más acentuada, llegando a valores cercanos a cero en el tratamiento que corresponde al mayor déficit hídrico (contenido de agua de $10 \%$ ) (figura 2A y B). Para la biomasa fresca y seca del sistema radical (figura 3A y B), así como en el volumen total de 
raíces (figura 3C), hubo un decrecimiento exponencial con el aumento del déficit hídrico.

Los resultados del índice de calidad de Dickson (ICD) mostraron una reducción exponencial con el aumento de los niveles de déficit hídrico, es decir, cuanto mayor fue la disponibilidad de agua (contenido de agua de $100 \%$ ), mayor fue el valor obtenido (figura 4A). En este sentido, el mayor valor medio fue de 5,37 en el tratamiento con el mayor régimen hídrico, presentando reducción a 0,7 en el $25 \%$ de contenido de agua.
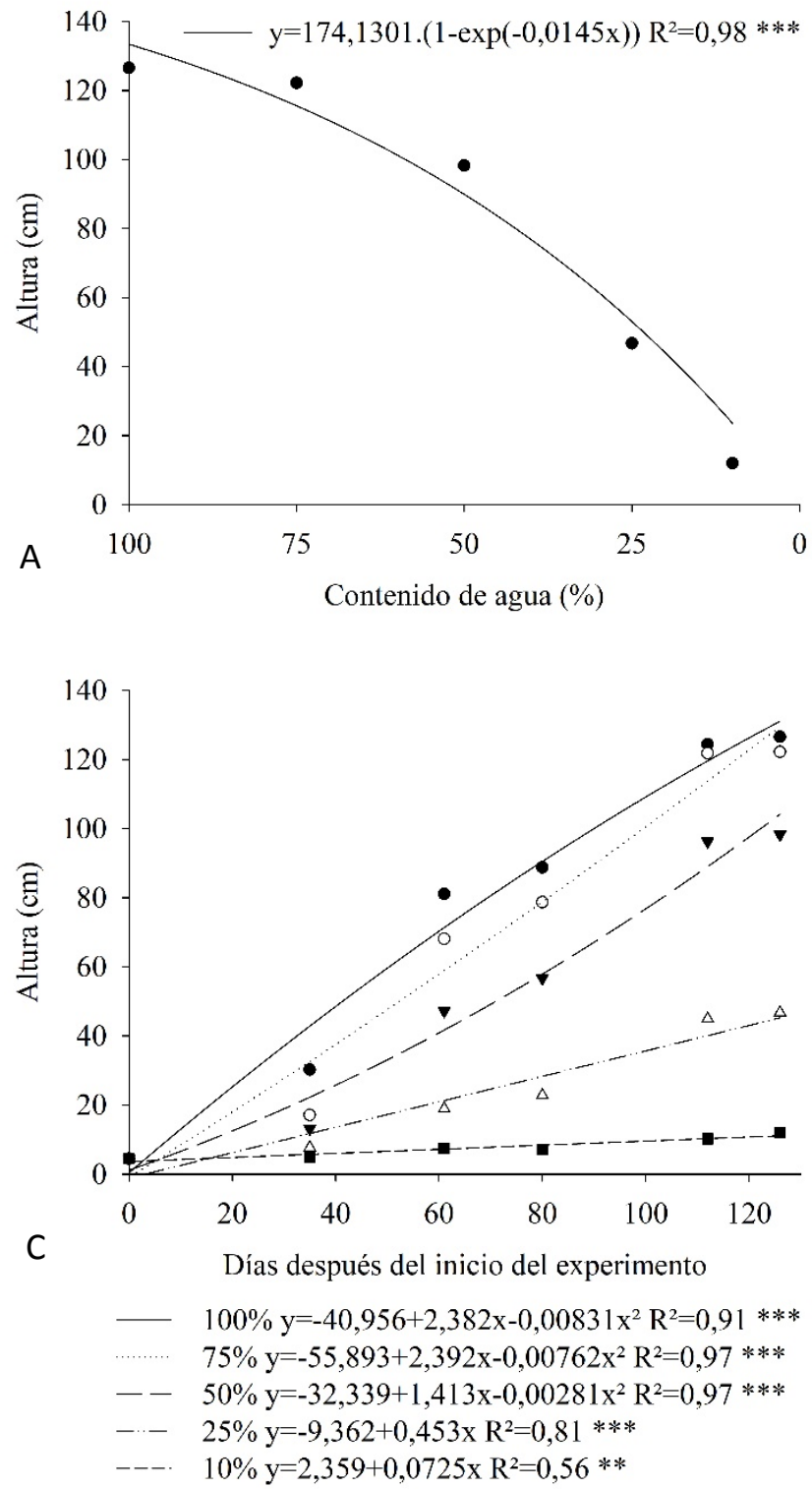

La relación de la altura con la masa seca de la parte aérea presentó una tendencia exponencial creciente con la reducción de la disponibilidad hídrica (figura 4B), mostrando que el crecimiento en altura fue mayor que el incremento en biomasa de la parte aérea a medida que disminuía la disponibilidad hídrica. En contrapartida, no hubo influencia de los niveles de régimen hídrico en la relación de la masa seca de la parte aérea con la masa seca del sistema radical (figura 4C), presentando media general de 2,14.
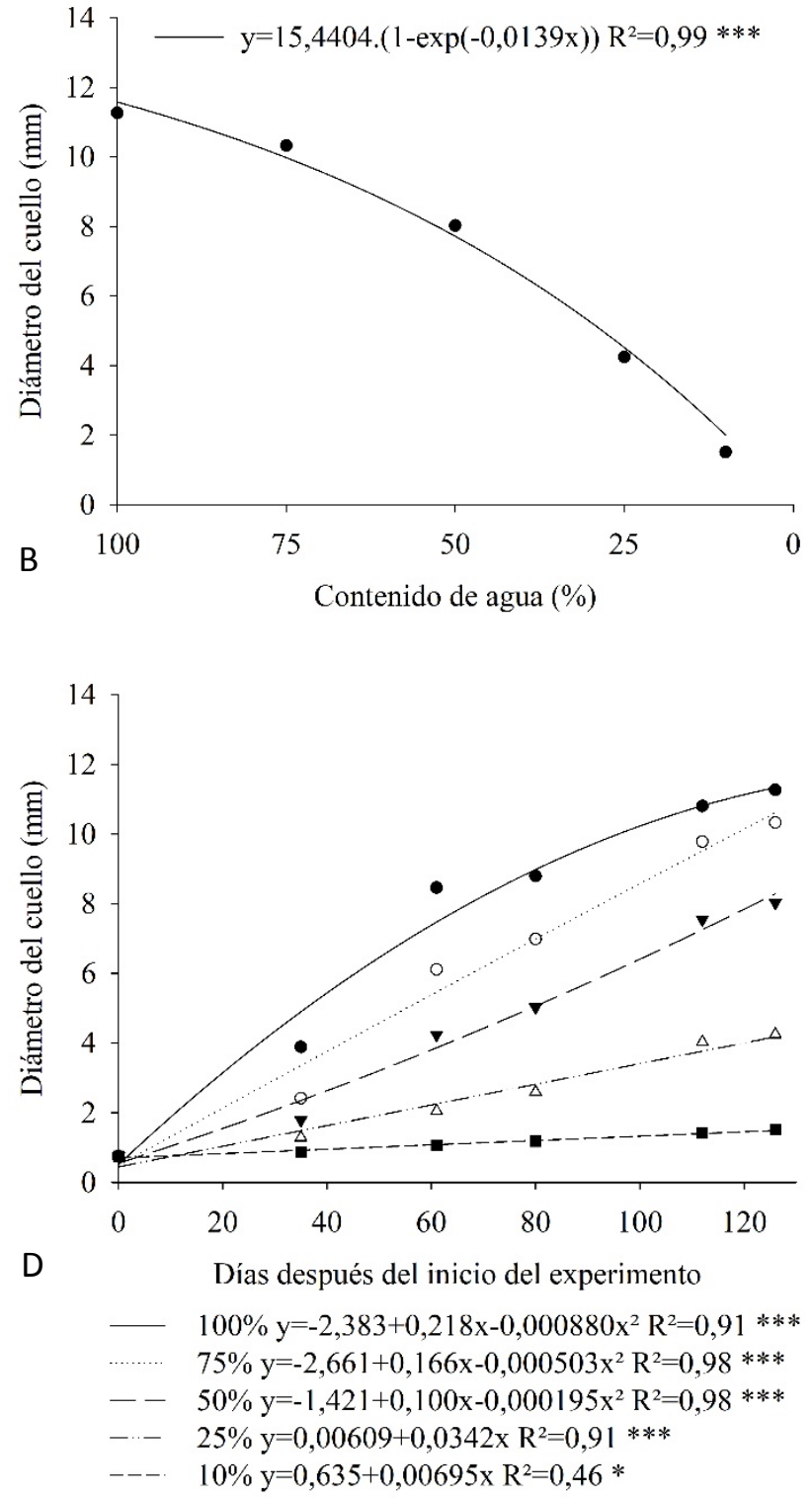

Figura 1. Altura (A) y diámetro del cuello (B) de plantines de Mimosa scabrella en función de diferentes regímenes hídricos, a los 126 días después de la instalación del experimento, y a lo largo del tiempo de conducción del experimento $(\mathrm{C}$ y $\mathrm{D})$. * $P<0,05 ; * * P$ $<0,01 ; * * * P<0,001$.

Height (A) and root-collar diameter (B) of Mimosa scabrella seedlings as a function of different water regimes, at 126 days after installation of the experiment, and throughout the experimentation time $(\mathrm{C}$ and $\mathrm{D}) . *=P<0.05 ; * *=P<0.01 ; * * *=P<0.001$. 

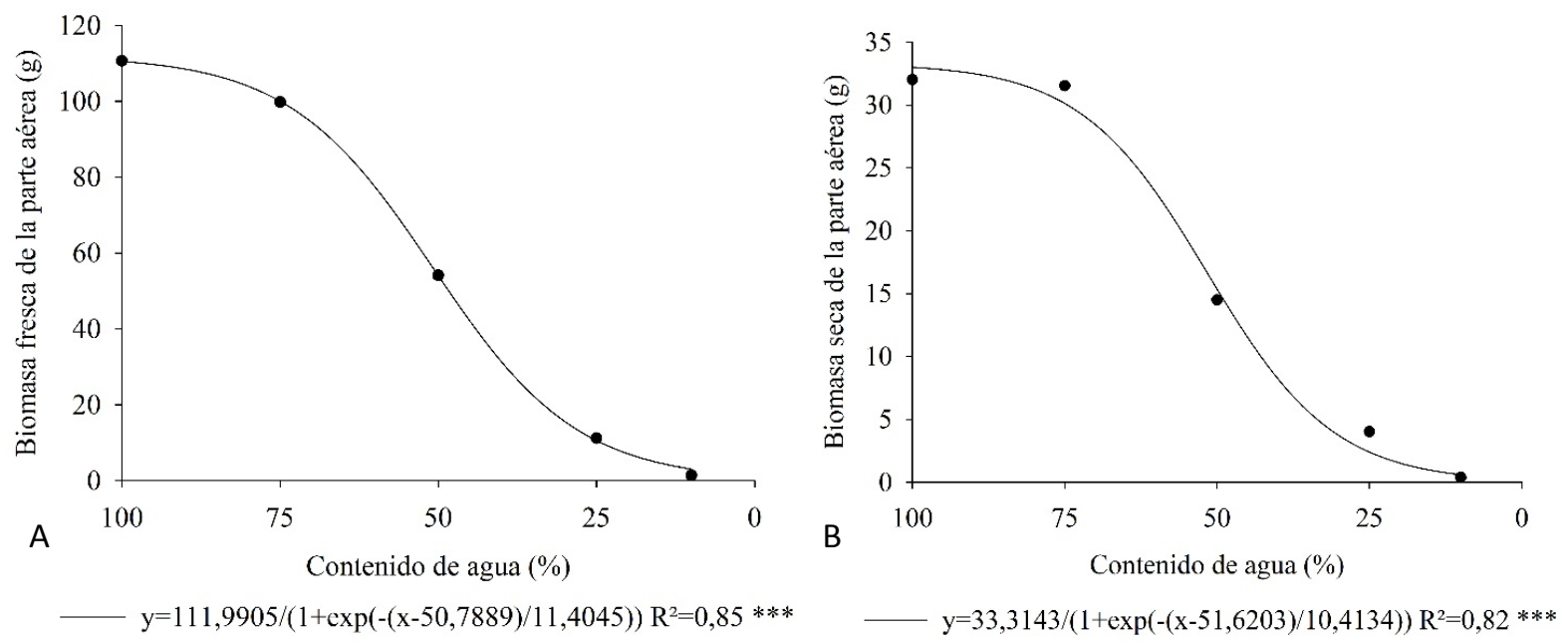

Figura 2. Biomasa fresca (A) y biomasa seca de la parte aérea (B) de plantines de Mimosa scabrella en función de diferentes regímenes hídricos, a los 126 días después de la instalación del experimento. $* * * P<0,001$.

Fresh (A) and dry biomass of the aerial part (B) of Mimosa scabrella seedlings as a function of different water regimes, at 126 days after installation of the experiment. $* * *=P<0.001$.
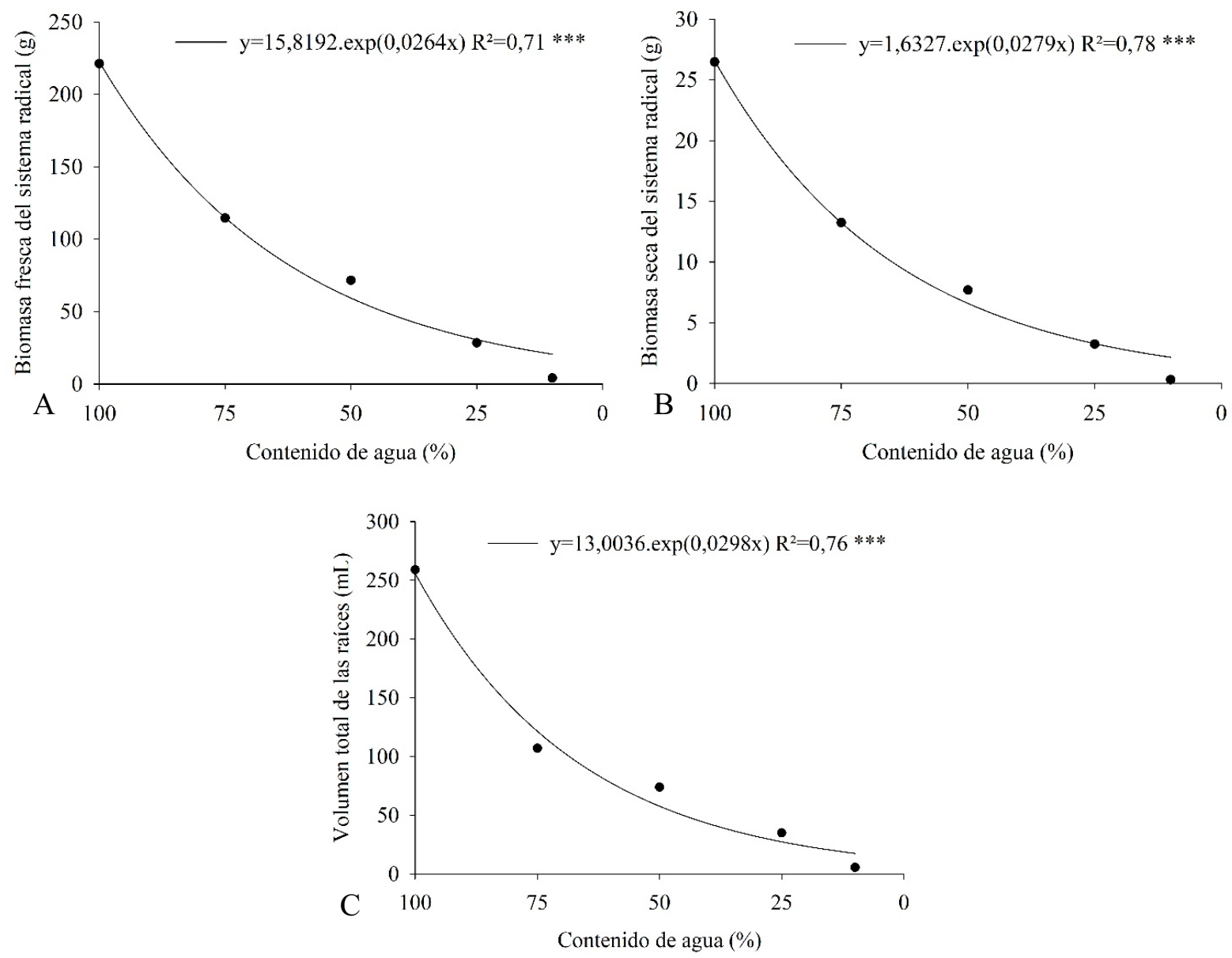

Figura 3. Biomasa fresca (A) y biomasa seca del sistema radical (B) y volumen total de raíces (C) de plantines de Mimosa scabrella en función de diferentes regímenes hídricos, a los 126 días después de la instalación del experimento. $* * * P<0,001$.

Fresh biomass (A) and dry matter of the root system (B) and total volume of roots of Mimosa scabrella seedlings as a function of different water regimes, 126 days after the installation of the experiment. $* * *=P<0.001$. 


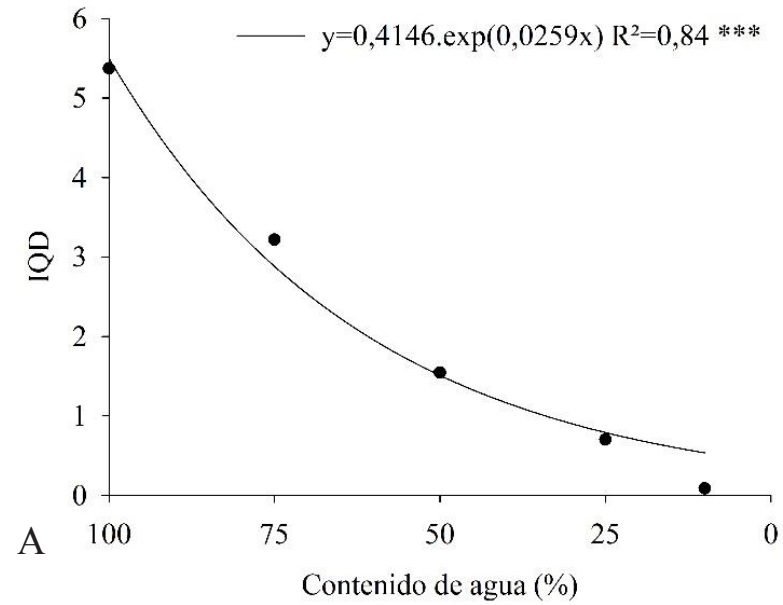

Contenido de agua $(\%)$
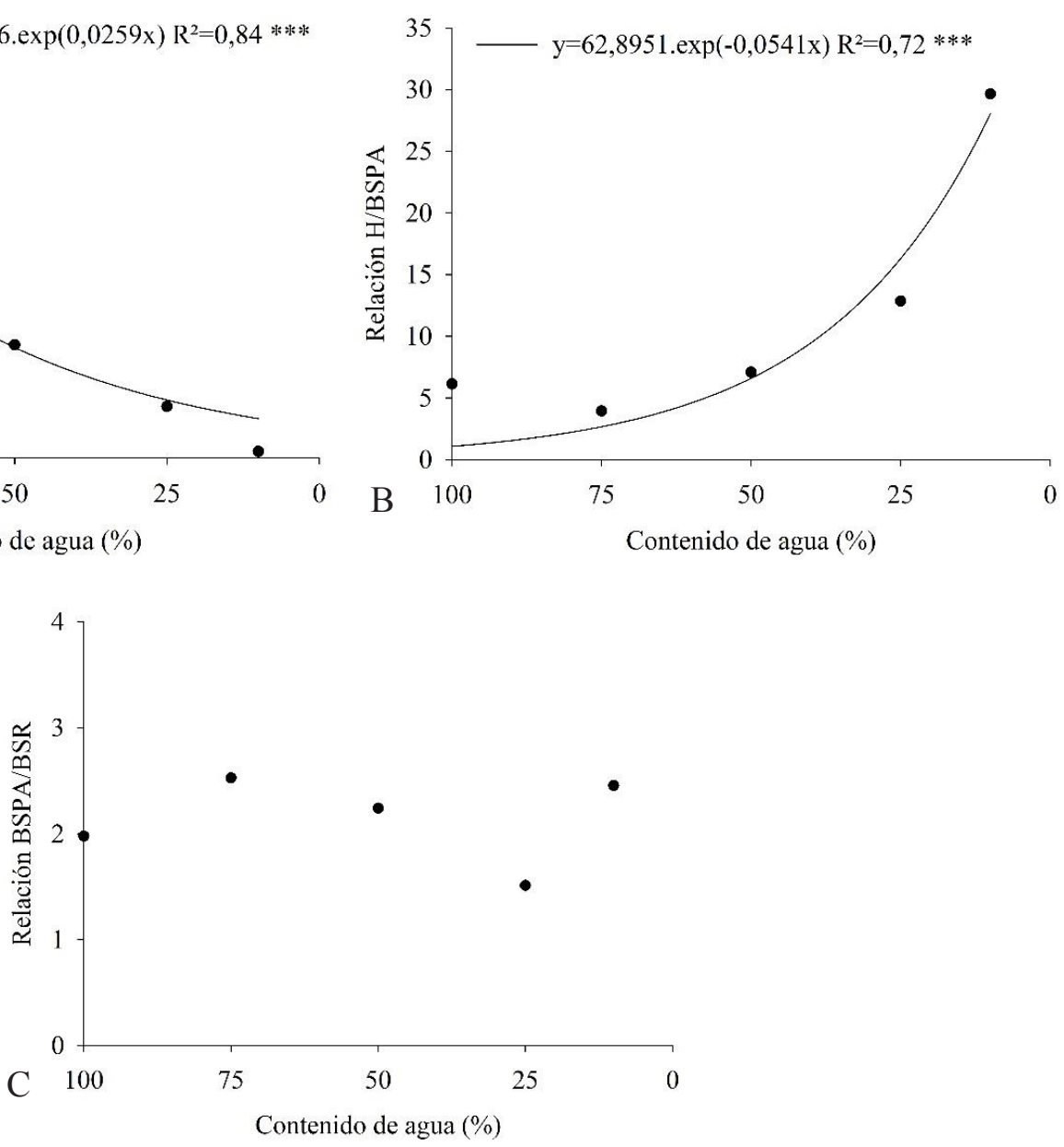

Figura 4. Índice de calidad de Dickson (ICD) (A), relación altura y biomasa seca de la parte aérea (H/BSPA) (B) y relación biomasa seca de la parte aérea y sistema radical (BSPA/BSR) (C) de plantines de Mimosa scabrella en función de diferentes regímenes hídricos, a los 126 días después de la instalación del experimento. *** $P<0,001$.

Dickson Quality Score (ICD) (A), relation height and dry mass of the aerial part (H/BSPA) (B) and dry biomass ratio of the aerial part and root system (BSPA/BSR) (C) of Mimosa scabrella seedlings as a function of different water regimes, 126 days after the installation of the experiment. $* * *=P<0.001$.

\section{DISCUSIÓN}

Los niveles de estrés hídrico incidieron de forma proporcional en el desarrollo inicial de los plantines de bracatinga, pues con la reducción de la disponibilidad hídrica hubo reducción en el crecimiento en las plantas. Resultados similares se han observado en plantas de Libidibia ferrea (Mart. ex Tul.) L.P. Queiroz (Lenhard et al. 2010) y Azadirachta indica A. Juss (Martins et al. 2010), donde hubo mayor altura de los brinzales cuando sometidas a la mayor disponibilidad hídrica. Cuando una planta es sometida al estrés hídrico tiene prácticamente todos los aspectos del crecimiento y desarrollo afectados (Achakzai 2009). Por lo tanto, el bajo desarrollo de los plantines de bracatinga en altura y diámetro del cuello, obtenidos con los mayores niveles de déficit hídrico, pueden ser explicados porque la reducción en la disponibilidad hídrica reduce el potencial hídrico (Verslues et al. 2006), lo que irá a afectar la presión de turgencia y, en consecuencia, el elongamiento y división celular (Soares et al. 2015). La tasa fotosintética también se ve afectada por la restricción en la absorción de $\mathrm{CO}_{2}$ debido al cierre estomático, y en muchos casos por efectos más directos en el metabolismo (Verslues et al. 2006).

Lo que puede explicar los menores resultados obtenidos para altura y diámetro del cuello a lo largo del tiempo de conducción del experimento en elevados niveles de deficiencia hídrica, es la sensibilidad de un determinado proceso fisiológico al déficit hídrico, lo que es un reflejo de la estrategia de la planta en lidiar con el rango de variación de la disponibilidad hídrica (Taiz y Zeiger 2013). De esta forma, la deficiencia hídrica puede afectar directamente el crecimiento en altura y diámetro del cuello, reduciendo la expansión celular y la formación de la pared celular. No 
obstante, efectos indirectos pueden estar relacionados con la reducción en la disponibilidad de carbohidratos o influenciando la producción de reguladores de crecimiento (Gonçalves y Passos 2000).

De acuerdo con Taiz y Zeiger (2013), la disminución de la biomasa fresca y seca de la parte aérea con el aumento del estrés hídrico puede estar relacionada con un balance funcional entre absorción de agua por la raíz y fotosíntesis por la parte aérea. Por lo tanto, una parte aérea tenderá a crecer hasta que la absorción de agua por las raíces se convierta en una limitante para el crecimiento, así como las raíces tenderán a crecer hasta que su demanda por fotosintatos provenientes de la parte aérea exceda el suministro. Cuando el agua para la parte aérea se vuelve limitante, la expansión foliar se reduce antes de que la actividad fotosintética se vea afectada.

En un estudio con plantas de pau-ferro (L. ferrea), se verificó una mayor acumulación de biomasa seca de la parte aérea cuando se sometieron al $40 \%$ de la capacidad de la maceta (Lenhard et al. 2010). Resultados similares se observaron en M. urundeuva (Figuero et al. 2004) y Tabebuia aurea (Silva Manso) Benth. et Hook.f. ex S.Moore (Cabral et al. 2004). Sin embargo, para diferentes genotipos de citrus, se verificó que la reducción en la lámina de agua aplicada en las plantas comprometió su crecimiento, tanto en la biomasa seca aérea y del sistema radical (Soares et al. 2015).

Diversos autores han demostrado que las plantas que crecen bajo déficit hídrico tienden a presentar mayor desarrollo radical, ya que necesitan absorber agua de capas más profundas del suelo, aumentando así la eficiencia en la captura de agua (Santos y Carlesso 1998, Lenhard et al. 2010). Sin embargo, los resultados obtenidos en este trabajo no siguen esta tendencia pues con el aumento del déficit hídrico hubo decrecimiento exponencial en la biomasa fresca y seca del sistema radical, así como en el volumen total de raíces.

Este descenso en la masa fresca y seca del sistema radical y en el volumen total de raíces con el aumento de los niveles de déficit hídrico puede estar relacionado con la baja cantidad de agua diaria que los tratamientos con menor contenido de agua recibieron en el momento del riego. De acuerdo con Taiz y Zeiger (2013), en la medida en que las células vegetales pierden agua, se encogen, principalmente debido al colapso de las paredes celulares. De esta forma, cuando las células reducen su tamaño ocurre también una reducción del órgano al cual ellas forman parte, en este caso las raíces. Cuando estas se contraen, la superficie de las raíces puede alejarse de las partículas del suelo que retiene el agua y es posible que los pelos absorbentes sean dañados. A pesar de que las raíces crecen lentamente durante el desecamiento, la capa externa de su corteza se cubre en mayor medida con suberina, un lípido impermeable al agua, además de aumentar la resistencia al flujo de agua hacia la raíz (Taiz y Zeiger 2013).

Los resultados verificados por Figuerôa et al. (2004) corroboran con el presente estudio, pues a los 60 días des- pués del inicio del experimento, los autores verificaron reducción en la masa seca del sistema radical de plantas de M. urundeuva con la reducción de la disponibilidad hídrica. Resultados similares fueron verificados por Cabral et al. (2004), donde a partir de 90 días después del inicio del experimento observaron una reducción de la biomasa seca del sistema radical de plantas de T. aurea, en el $25 \%$ de la capacidad de la maceta, y por Martins et al. (2010) que también verificaron una reducción de la biomasa seca del sistema radical de plantas de $A$. indica con la disminución de la disponibilidad hídrica.

El patrón de calidad de los brinzales está directamente relacionado con la supervivencia, el establecimiento y el crecimiento inicial de los bosques, y por eso es de fundamental importancia la evaluación de estos parámetros. El índice de calidad de Dickson (ICD) es un buen indicador de la calidad de los plantines, pues tiene en cuenta la robustez y el equilibrio de la distribución de biomasa de la planta. Además, otros importantes parámetros para la evaluación de la calidad de las plantas son la relación de la altura con la biomasa seca de la parte aérea (H/BSPA) y la relación de la biomasa seca de la parte aérea con la biomasa seca del sistema radical (BSPA/BSR), donde el primero se utiliza para predecir el potencial de supervivencia de los brinzales en el campo y el segundo se utiliza para mediciones de crecimiento en estudios ecológicos y fisiológicos (Gomes y Paiva 2013).

Con base en trabajos de investigación, se recomienda el valor mínimo de 0,20 para el índice de calidad de Dickson, como un buen indicador para la calidad de plantas de Pseudotsuga menziesii (Mirb.) Franco y Picea abies (L.) Karst (Gomes y Paiva 2013). Además, para las plantas de Eucalyptus grandis Hill ex Maiden con 120 días desde la germinación se obtuvo el valor de aproximadamente 0,07 (Rudek et al. 2013), mientras que para Senna macranthera (dc. Ex Collad.) H. S. Irwin et Barneby el valor promedio fue de 1,41 (Chaves y Paiva 2004). Así, se verifica que, incluso ocurriendo una reducción en el ICD con el aumento del déficit hídrico, las plantas presentan una calidad para desarrollarse en ambiente con baja disponibilidad hídrica.

Según Gomes et al. (2002) la relación H/BSPA presentó mayor contribución relativa a la calidad de plantas de E. grandis, porque cuanto menor es este índice, más dura será la muda y mayor deberá ser su capacidad de supervivencia en el campo (Gomes y Paiva 2013). De esta manera, los resultados obtenidos en este estudio indican que la bracatinga presenta capacidad de sobrevivir en ambiente con restricción hídrica, pues aun ocurriendo un aumento de esta relación con la disminución en la disponibilidad hídrica, el menor valor obtenido fue en el tratamiento con el $75 \%$ de contenido de agua $(3,95)$, además de los resultados similares comprobados del tratamiento control hasta el $25 \%$ de contenido de agua.

En muchas plantas las reducciones de la disponibilidad hídrica inhiben el crecimiento del tallo y la expansión celular, pero estimulan el crecimiento de las raíces, lo que 
puede considerarse una respuesta adecuada a las condiciones de déficit hídrico (Taiz y Zeiger 2013). Sin embargo, las plantas no necesariamente necesitan ser resistentes a la sequía para vivir en regiones con deficiencia hídrica, pues hay especies que programan su crecimiento para períodos con suficiente disponibilidad hídrica (Larcher 2000). Esto podría explicar que las plantas de bracatinga no invirtieran en una mayor formación del sistema radical en niveles de alta deficiencia hídrica, ya que no hubo influencia de los niveles de régimen hídrico en la relación de la biomasa seca de la parte aérea con la biomasa seca del sistema radical. No obstante, de acuerdo con Gomes y Paiva (2013) la mejor relación entre BSPA/BSR es de 2,0, lo que confirma los resultados satisfactorios obtenidos para la bracatinga, ya que la media general fue de 2,14.

La supervivencia de plantas sometidas al estrés hídrico será mayor mientras más tiempo se tarde en ocurrir la disminución del potencial hídrico y mientras mayor sea la capacidad del protoplasma de tolerar la desecación sin presentar daños (Larcher 2000). El elevado porcentaje de supervivencia de cinco especies del género Acacia en condiciones de estrés hídrico indican que estos genotipos pueden soportar esta condición durante el establecimiento de sus plantas (Venier et al. 2013). Respecto a esto, se resalta la posibilidad de la utilización de la bracatinga bajo déficit hídrico, pues del total de plantas probadas, hubo mortalidad de solo tres individuos que estaban sometidos al menor nivel de disponibilidad hídrica (10\% de contenido de agua).

El estrés hídrico, en general, conduce a una acumulación de solutos en el citoplasma y en la vacuola de las células vegetales, permitiendo así que las células mantengan la presión de turgencia incluso en un bajo potencial hídrico. Esta presión de turgencia positiva es fundamental para el crecimiento vegetal, así como aumentar la rigidez mecánica de células y tejidos (Smirnoff 2014). Además, es importante para permitir la continuidad del crecimiento, expansión, división celular y fotosíntesis (Santos y Carlesso 1998), lo que puede explicar los resultados obtenidos en este estudio en plantas sometidas a bajos niveles de disponibilidad hídrica.

Cuando comienza a disminuir la turgencia de las células, se inician medidas osmoregulatorias a través de la acumulación de sustancias orgánicas de bajo peso molecular en los compartimentos celulares y en el citosol, promoviendo así el influjo de agua. Estas medidas ayudan al mantenimiento del volumen celular, retardando la pérdida de turgencia, manteniendo así por un tiempo mayor la apertura estomática (Larcher 2000). En este contexto, es necesario verificar si las plantas de bracatinga realizan el ajuste osmótico para mantener su crecimiento en condiciones de deficiencia hídrica.

De acuerdo con Larcher (2000), la fijación de nitrógeno en los nódulos de las raíces de las especies leguminosas es muy sensible a la sequía. Sin embargo, para Engin y Sprent (1973) la severidad de esta respuesta varía con el grado y la duración del estrés. En el estudio con Phaseolus vulgaris L., el potencial de fijación biológica de nitrógeno, evaluado por el número y peso seco de nódulos, fue poco afectado por el estrés hídrico (Pimentel y Perez 2000). No obstante, se observó que el efecto del déficit hídrico puede ser minimizado por la presencia de micorrizas arbusculares, pues la actividad de los nódulos fue significativamente mayor en plantas inoculadas con relación a las sin inoculación (Peña et al. 1988). Por lo tanto, hay necesidad de estudiar el desarrollo de plantas de bracatinga en condiciones simbióticas, a fin de verificar si esos resultados se confirman en esas condiciones, pues en este estudio no se inocularon cepas de bacterias fijadoras de nitrógeno y por eso no se encontraron nódulos en las raíces.

En general, los resultados obtenidos en este estudio sugieren que la bracatinga puede ser utilizada en planes de manejo y restauración de áreas, así como su producción comercial en áreas con estrés hídrico, pues hasta niveles intermediarios de deficiencia hídrica no hubo decrecimiento acentuado en el crecimiento y la calidad de los brinzales se presentó satisfactoria. Sin embargo, ello debe verificarse y validarse en campo.

\section{CONCLUSIONES}

Los resultados muestran una buena respuesta de $M$. scabrella a períodos de estrés hídrico, lo que sugiere que la especie pueda ocupar sitios con menor disponibilidad de agua, establecerse temprano en la sucesión ecológica o permanecer en el tiempo ante una restricción del recurso, pues hasta el $50 \%$ de contenido de agua el crecimiento y el desarrollo inicial de las plantas presentan resultados satisfactorios. Además, las plantas presentan capacidad de sobrevivir y establecerse en condiciones de estrés hídrico.

\section{AGRADECIMIENTOS}

Los autores agradecen la Coordinación de Perfeccionamiento de Personal de Nivel Superior (CAPES) y la Fundación de Amparo a la Investigación del Estado de Rio Grande do Sul (FAPERGS).

\section{REFERENCIAS}

Achakzai AKK. 2009. Effect of water stress on imbibition, germination and seedling growth of maize cultivars. Sarhad Journal of Agriculture 25(2): 165-172.

Cabral EL, DCA Barbosa, EA Simabukuro. 2004. Crescimento de plantas jovens de Tabebuia aurea (Manso) Benth. et Hook. f. ex S. Moore submetidas a estresse hídrico. Acta Botanica Brasilica 18(2): 241-251. DOI: dx.doi.org/10.1590/S010233062004000200004

Chaves AS, HN Paiva. 2004. Influência de diferentes períodos de sombreamento sobre a qualidade de plantines de fedegoso (Senna macranthera (Collad.) Irwin et Barn.). Scientia Forestalis $65:$ 22-29.

Claeys H, D Inzé. 2013 The agony of choice: how plants balance 
growth and survival under water-limiting conditions. Plant Physiology 162(4): 1768-1779. DOI: doi.org/10.1104/ pp.113.220921

Dickson A, AL Leaf, JF Hosner. 1960. Quality appraisal of white spruce and white pine seedling stock in nurseries. Forestry Chronicle 36(1): 10-13. DOI: doi.org/10.5558/tfc36010-1

Engin M, JI Sprent. 1973. Effects of water stress on growth and nitrogen-fixing activity of Trifolium repens. New Phytologist 72: 117-126. DOI: doi.org/10.1111/j.1469-8137.1973. tb02016.x

Fabrowski FJ, GIB Muñiz, MCM Mazza, T Nakashima, U Klock, JC Possamai, S Nisgoski. 2005. Anatomia comparativa da madeira das variedades populares da bracatinga (Mimosa scabrella Bentham). Ciência Florestal 15(1): 65-73. DOI: dx.doi.org/10.5902/198050981824

Gomes JM, HN Paiva. 2013. Viveiros florestais: propagação sexuada. Viçosa, Brasil. Universidade Federal de Viçosa. $116 \mathrm{p}$.

Gomes JM, L Couto, HG Leite, A Xavier, SLR Garcia. 2002. Parâmetros morfológicos na avaliação da qualidade de plantines de Eucalyptus grandis. Revista Árvore 26(6): 655664. DOI: dx.doi.org/10.1590/S0100-67622002000600002

Gonçalves MR, CAM Passos. 2000. Crescimento de cinco espécies de eucalipto submetidas a déficit hídrico em dois níveis de fósforo. Ciência Florestal 10(2): 145-161. DOI: dx.doi. org/10.5902/19805098488

Hoffer H, AB Debastiani, RP Martins Neto, RD Menegatti, SL Rafaeli Neto. 2015. Variabilidade espacial do estado de compactação do solo em um sítio experimental de Mimosa scabrella Benth. Enciclopédia Biosfera 11(21): 1903-1913.

Larcher W. 2000. Ecofisiologia vegetal. São Carlos, Brasil. RiMa. 531 p.

Lenhard NR, SPQ Scalon, JO Novelino. 2010. Crescimento inicial de plantines de pau ferro (Caesalpinia ferrea Mart. ex Tul. var. leiostachya Benth.) sob diferentes regimes hídricos. Ciência e Agrotecnologia 34(4): 870-877. DOI: dx.doi. org/10.1590/S1413-70542010000400011

Lopes JLW, IA Guerrini, MR Silva, JCC Saad, CF Lopes. 2011. Estresse hídrico em plantio de Eucalyptus grandis vs. Eucalyptus urophylla, em função do solo, substrato e manejo hídrico de viveiro. Revista Árvore 35(1): 31-39. DOI: dx.doi.org/10.1590/S0100-67622011000100004

Machado AS, ACPS Bartoszeck, A Figueiredo Filho, EB Oliveira. 2008. Efeito da densidade e do sítio sobre as curvas de distribuição diamétrica em bracatingais nativos (Mimosa scabrella Benth.) da região metropolitana de Curitiba. Ambiência 4(1): 37-50.

Martins MO, RJMC Nogueira, AD Azevedo Neto, MG Santos. 2010. Crescimento de plantas jovens de nim-indiano ( $\mathrm{Aza}$ dirachta indica A. Juss. - Meliaceae) sob diferentes regimes hídricos. Revista Árvore 34(5): 771-779. DOI: dx.doi. org/10.1590/S0100-67622010000500002

Menegatti RD, A Mantovani, MC Navroski, AG Souza. 2017.
Genetic divergence among provenances of Mimosa scabrella Benth. based on seed analysis. Revista Brasileira de Ciências Agrárias 12(3): 366-371. DOI: 10.5039/agraria. v12i3a5449

Menegatti RD, A Mantovani, MC Navroski. 2016. Parâmetros genéticos para caracteres de crescimento inicial de progênies de bracatinga em Lages, SC. Pesquisa Florestal Brasileira 36(87): 235-243. DOI: dx.doi.org/10.4336/2016. pfb.36.87.1003

Peña JI, M Sánchez-Díaz, J Aguirreolea, M Becana. 1988. Increased stress tolerance of nodule activity in the Medicago-Rhizobium-Glomus symbiosis under drought. Journal of Plant Physiology 133(1): 79-83. DOI: doi.org/10.1016/ $\underline{\mathrm{S} 0176-1617(88) 80088-9}$

Pimentel C, AJC Perez. 2000. Estabelecimento de parâmetros para avaliação de tolerância à seca, em genótipos de feijoeiro. Pesquisa Agropecuária Brasileira 35(1): 31-39. DOI: dx.doi.org/10.1590/S0100-204X2000000100005

Rudek A, FAO Garcia, FSB Peres. 2013. Avaliação da qualidade de mudas de eucalipto pela mensuração da área foliar com o uso de imagens digitais. Enciclopédia Biosfera 9(17): 3775-3787.

Santos RF, R Carlesso. 1998. Déficit hídrico e os processos morfológico e fisiológico das plantas. Revista Brasileira de Engenharia Agrícola e Ambiental 2(3): 287-294. DOI: dx.doi. org/10.1590/1807-1929/agriambi.v2n3p287-294

Saueressig D. 2014. Plantas do Brasil: árvores nativas. Irati, Brasil. Editora Plantas do Brasil. 432 p.

Silva MBR, RA Viégas, J Dantas Neto, SAR Farias. 2009. Estresse salino em plantas da espécie florestal sabiá. Caminhos de Geografia 10(30): 120-127.

Smirnoff N. 2014. Plant stress physiology. Chichester, UK. John Wiley. DOI: doi.org/10.1002/9780470015902.a0001297. pub2

Soares LAA, MEB Brito, PD Fernandes, GS Lima, WS Soares Filho, ES Oliveira. 2015. Crescimento de combinações copa - porta-enxerto de citros sob estresse hídrico em casa de vegetação. Revista Brasileira de Engenharia Agrícola e Ambiental 19(3): 211-217. DOI: dx.doi.org/10.1590/18071929/agriambi.v19n3p211-217

Taiz L, E Zeiger. 2013. Fisiologia vegetal. Porto Alegre, Brasil. Artmed. $918 \mathrm{p}$.

Venier P, M Cabido, A Mangeaud, G Funes. 2013. Crecimiento y supervivencia de plántulas de cinco especies de Acacia (Fabaceae), que coexisten en bosques secos neotropicales de Argentina, en distintas condiciones de disponibilidad de luz y agua. Revista de Biologia Tropical 61(2): 501-514. DOI: $\underline{10.15517 / \text { rbt.v61i2.11143 }}$

Verslues PE, M Agarwal, S Katiyar-Agarwal, J Zhu. 2006. Methods and concepts in quantifying resistance to drought, salt and freezing, abiotic stresses that affect plant water status. The Plant Journal 45(6): 523-539. DOI: doi. org/10.1111/j.1365-313X.2005.02593.X 
